\title{
Research on the Modified Model for Steady Displacement Interface Shape in Inclined Wells with Eccentric Annuli
}

\author{
Feng Fuping*, Yu Fahao, Ai Chi, Cui Zhihua and Xu Haisu
}

Institute of Petroleum Engineering, Northeast Petroleum University, Daqing 163318, China

\begin{abstract}
It is well known that a displacement interface reflects the intermixing extent of two-phase fluids. Optimization of displacing and fluids' property parameters is the key to achieve good cementing quality according to the displacement interface shape. In previous literatures, the model for the steady displacement interface shape in inclined wells only takes the factor of annular azimuthal angle into account, but not including annular radium term, which is imperfect. In this paper, by comprehensively analyzing the effects of both azimuthal angle and annular radium on the displacement interface shape, we establish a modified model for the steady displacement interface shape in inclined wells with eccentric annuli on the basis of a 2D Hele-Shaw displacement model, which will be more suitable for describing the actual displacement interface shape. From the modified model, we obtain: When density difference is smaller, the position of interface front is on the external casing wall of the annular wide side; otherwise, it will occur on the wellbore wall of the annular narrow side under a greater density difference. The displacement interface length in inclined wells with eccentric annuli is decreased and then increased with the increase of density difference, which indicates that there does exist an optimal density difference to make the interface length minimum. For a certain casing's eccentricity, the optimal density difference in inclined wells is gradually decreased with deviation angle. When meeting the conditions of pressure stability and anti-leak, a greater density difference can obtain better displacement effect for vertical wells, otherwise, a smaller density difference can be good for horizontal wells.
\end{abstract}

Keywords: Inclined wells, casing's eccentricity, displacement interface, steady displacement, Hele-Shaw displacement model.

\section{INTRODUCTION}

A displacement interface reflects the intermixing extent of two-phase fluids in cement. A longer interface means that more displacing fluids become contaminated. When the interface length gets out of control, that is to say, maintains growth, the accident that the interface trailing edge cannot return out the cementing segment at the end of cement operation may happen, as a result, which decreases the displacing efficiency $[1,2]$. There is no doubt that a bad annular cementing quality can lead to any of the following: blowout, leakage at surface, destruction of subsurface ecology, potential contamination of freshwater, delayed or prevented abandonment, as well as loss of revenue. Therefore, decreasing the interface length is the premise to enhance the cementing quality.

For inclined wells, the displacement interface becomes more complex because of its unique loading conditions: (1) Casing tends to move toward the wellbore low side due to its own gravity, which creates a narrow annular clearance. Because of the greater resistance for fluid flow through the narrow clearance, it is easier for slurry to advance through the wide clearance on the wellbore up side. As a result, the interface will become longer and more complex; (2) There always exists a density difference between cement slurry and drilling mud. Heavy slurry tends to flow toward the wellbore

*Address correspondence to this author at the No.199 FaZhan Road, HighTech Development Zone, Daqing 163318, China; Tel: 13904696932;

Fax: 0459-6503482; E-mail: fengfuping2005@163.com low side and light drilling mud always gathers in the up side, leading to the advancement of cement slurry in the wellbore up side and drilling-mud retention in the low side. Analysis on the two reasons, we can find that the effects of casing's eccentricity and fluids' density differences on the displacement interface are opposite, which is different with vertical wells $[3,4]$.

S.H. Bittleston et al. divided the displacement situations into steady displacement, unsteady displacement and drilling fluid channel [5-13] (which was verified by S. Malekmohammadi in laboratory experiments [14]). Besides, they established a calculation model for the displacement interface shape. Unfortunately, the calculation model was not perfect. It only took the effect of annular azimuthal angle on the interface shape into consideration, but not including annular radius. They thought the interface at different radium had the same shape for a certain azimuthal angle. The theory was suitable for the vertical well. However, for inclined wells, because of the driving force generated by the density difference between slurry and drilling fluid depending on fluids' positions, the driving force was a function of azimuthal angle and annular radius, the interface shape changed with annular radium could not be neglected.

In this paper, on the basis of a Hele-Shaw displacement model, we establish a modified model for the steady displacement interface shape in inclined wells with eccentric annuli which includes the annular radium term. The modified model lays a good foundation for analyzing the effects of density difference and deviation angle on interface 


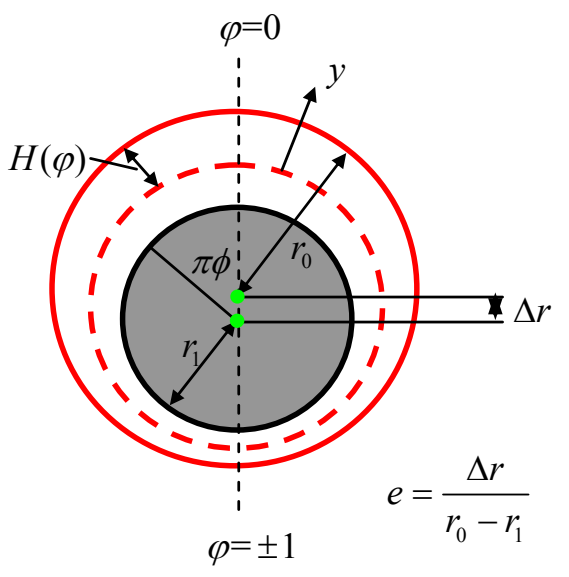

Fig. (1). Hele-Shaw physical model for cementing displacement.

shape, besides, provides a basis for the optimization of displacing parameters and fluids' property parameters during inclined well cementing.

\section{THE MODIFIED HELE-SHAW MODEL IN INCLINED WELLS WITH ECCENREIC ANNULI}

\subsection{Hele-Shaw Physical Model for Cementing Displacements}

A Hele-Shaw modeling approach derived by S.H. Bittleston et al. in 2002 is appropriate when analyzing cementing displacement on the scale of annular gap. We consider a simplified version of the model. The simplification that we make is that the annulus is locally uniform in the axial direction, i.e., over a length scale that is long in comparison to the azimuthal scale.

Dimensionless spatial coordinates are $(\varphi, \xi) \in(0,1) \times(0$, $Z$ ). Here, $\varphi$ is the azimuthal coordinate with $\varphi=0$ denoting the wide side of the annulus and $\varphi=1$ the narrow (lower) side. The flow is assumed symmetric about $\varphi=0$, and thus only half the annulus is considered. This assumption implies that the narrow side of the annulus is always lying on the lower side of the well. The $\xi$-coordinate measures axial depth upwards along the annulus, as shown in Fig. (1). Here, $Z$ denotes the length of the section of well to be cemented and typical lengths of cemented sections are of the order of hundreds of meters.

For the process of slurry displacing drilling mud in an inclined wells with eccentric annuli, so as to simplify the theoretical model deduction, we assume that:

- Slurry and drilling fluid are characterized as Herschel-Bulkley fluids.

- The wellbore is a regular circle shape and its radium is the same at each azimuthal angle.

- $\quad$ Two-dimensional flow only along axial and azimuthal directions is considered.

- $\quad$ Slurry has a direct contact with drilling fluid between which there is no spacer fluid.
- The weight of the casing acts in such a way that the narrow side of the annulus will be found on the lower side of the well.

- Steady displacement can be obtained under our chosen slurry's performance parameters.

\subsection{The Modified Hele-Shaw Model for Inclined Well Cementing Displacements}

\subsection{1. $\mathrm{N}-\mathrm{S}$ Equation for Fluids on the Displacement Interface}

During cementing displacements, the force of slurry displacing drilling fluid includes the pressure $p^{*}$ which is provided by pumps lying on oilfield surface and the driving force caused by the density difference. For vertical wells, it is obviously seen that the density difference force is equal in a wellbore cross section because there is no plumb altitude difference. However, for inclined wells, the size of density difference force depends on the fluids' positions in the wellbore cross section, i.e., the plumb altitude from the wellbore up wall where pressure zero is. It is certain that the altitude changes with azimuthal angle and annular radium. Unfortunately, the Hele-Shaw model derived by S.H. Bittleston et al. is not successfully taken into account. So, a modified Hele-Shaw model during inclined well cementing is needed.

As shown in Fig. (2), the plumb altitude of $A$ from the wellbore up wall (pressure zero) in an inclined wellbore cross section is given by:

$h^{*}=\left(r_{0}^{*}-r^{*} \cos \pi \varphi\right) \sin a$

The azimuthal driving force caused by density difference is given by:

$$
M_{\varphi}^{*}=\left(\rho_{1}^{*}-\rho_{2}^{*}\right) g^{*}\left(r_{0}^{*}-r^{*} \cos \pi \varphi\right) \sin a
$$

The axial driving force caused by density difference is given by:

$$
M_{\xi}^{*}=\left(\rho_{1}^{*}-\rho_{2}^{*}\right)\left(r_{0}^{*}-r^{*} \cos \pi \varphi\right) g^{*} \cos a
$$




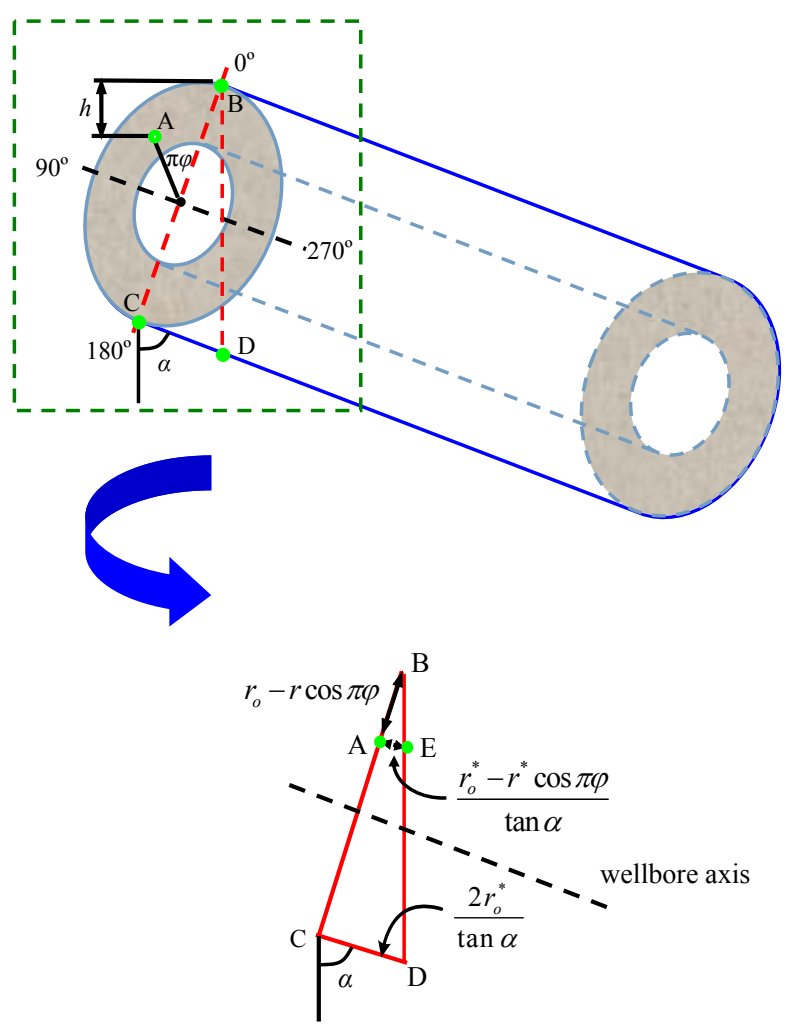

Fig. (2). The driving force caused by density difference in the inclined well annulus.

The gradient of azimuthal driving force in the $\varphi$ direction is equal to the partial derivative of $M_{\varphi}$ for $\varphi$, which is given by:

$$
\begin{aligned}
\frac{\partial M_{\varphi}^{*}}{\partial \varphi} & =\frac{\partial\left[\left(\rho_{1}^{*}-\rho_{2}^{*}\right) g^{*}\left(r_{0}^{*}-r^{*} \cos \pi \varphi\right) \sin a\right]}{\partial \varphi} \\
& =\pi\left(\rho_{1}^{*}-\rho_{2}^{*}\right) \sin \pi \varphi \sin a
\end{aligned}
$$

$A E$ is length derived from $r$ projecting in axial direction. $C D$ is a reference length which represents the deviation extent of displacement interface from vertical direction. If deviation angle $a$ is certain, the reference length is unchangeable. So, we take the ratio of $A E$ to $C D$ as the gradient of axial driving force, which is given by:

$$
\begin{aligned}
\frac{\partial M_{\xi}^{*}}{\partial \xi} & =\frac{\partial\left[\left(\rho_{1}^{*}-\rho_{2}^{*}\right)\left(r_{0}^{*}-r^{*} \cos \pi \varphi\right) g^{*} \cos a\right]}{\partial \xi} \\
& =\left(\rho_{1}^{*}-\rho_{2}^{*}\right) g^{*} \cos a \frac{A E}{C D}=\frac{\left(\rho_{1}^{*}-\rho_{2}^{*}\right)\left(r_{0}^{*}-r^{*} \cos \pi \varphi\right) g^{*} \cos a}{2 r_{o}}
\end{aligned}
$$

where $M_{\varphi}^{*}$ and $M_{\xi}^{*}$ represent the azimuthal and axial driving force caused by density difference respectively, MPa. $\rho_{1}^{*}$ and $\rho_{2}^{*}$ represent the actual density of slurry and drilling fluid respectively, $\mathrm{g} / \mathrm{cm}^{3} \cdot g^{*}$ is the gravitational acceleration, $9.81 \mathrm{~m} / \mathrm{s}^{2} . r_{0}^{*}$ is the actual wellbore radium, $\mathrm{m}$. $r^{*}$ represents the actual distance of any point $A$ on the displacement interface from wellbore center, $\mathrm{m} . \pi \varphi$ is the azimuthal angle, rad. $a$ is the deviation angle, ${ }^{\circ}$.

After mechanism analysis of fluids on the displacement interface, we can obtain the dimensionless $\mathrm{N}-\mathrm{S}$ equation as follows:

$$
\left\{\begin{array}{l}
-\frac{\partial p}{\partial y}=0 \\
-\frac{1}{r_{a}} \frac{\partial p}{\partial \varphi}+\frac{\partial \tau_{\varphi, y}}{\partial y}+\frac{\pi \Delta \rho r \sin \pi \varphi \sin a}{S t^{*}}=0 \\
-\frac{\partial p}{\partial \xi}+\frac{\partial \tau_{\xi, y}}{\partial y}-\frac{\Delta \rho\left(r_{o}-r \cos \pi \varphi\right) \cos a}{2 r_{o} \cdot S t^{*}}=0
\end{array}\right.
$$

\subsubsection{Deviation of $\vec{S}$}

In the Hele-Shaw physical model (Fig. 1), we assume that slurry and drilling fluid are characterized as HerschelBulkley fluids. Also, the motion of fluids on the displacement interface satisfies N-S equation. So, we can obtain:

$$
\begin{aligned}
& \frac{\partial}{\partial y}\left[\eta \frac{\partial v_{s}}{\partial y}\right]=\frac{1}{r_{a}} \frac{\partial p}{\partial \varphi}-\frac{\pi \Delta \rho r \sin \pi \varphi \sin a}{S t^{*}} \\
& \frac{\partial}{\partial y}\left[\eta \frac{\partial w_{s}}{\partial y}\right]=\frac{\partial p}{\partial \xi}+\frac{\Delta \rho\left(r_{o}-r \cos \pi \varphi\right) \cos a}{2 r_{o} \cdot S t^{*}}
\end{aligned}
$$

The flows we consider are therefore two-dimensional and the flow variables have been averaged across the annular gap $H(\varphi, \xi)$. Using conditions of no-slip at the annulus walls to get the continuity equation:

$$
\frac{\partial}{\partial \varphi}[H \bar{v}]+\frac{\partial}{\partial \xi}\left[H r_{a} \bar{w}\right]=0
$$

According to the continuity equation (9), the average velocities in the azimuthal and axial directions can be expressed respectively by:

$$
\bar{v}=\frac{1}{H} \int_{0}^{H} v_{s} d y, \quad \bar{w}=\frac{1}{H} \int_{0}^{H} w_{s} d y
$$

By integrating (7) and (8) three times on $y$, we get:

$$
\begin{aligned}
& \bar{v}=-\left[\frac{1}{r_{a}} \frac{\partial p}{\partial \varphi}-\frac{\pi \Delta \rho r \sin \pi \varphi \sin a}{S t^{*}}\right] \frac{1}{H} \int_{0}^{H} \int_{y}^{H} \frac{\tilde{y}}{\eta(\tilde{y})} \mathrm{d} \tilde{y} \mathrm{~d} y \\
& \bar{w}=-\left[\frac{\partial p}{\partial \xi}+\frac{\Delta \rho\left(r_{o}-r \cos \pi \varphi\right) \cos a}{2 r_{o} \cdot S t^{*}}\right] \frac{1}{H} \int_{0}^{H} \int_{y}^{H} \frac{\tilde{y}}{\eta(\tilde{y})} \mathrm{d} \tilde{y} \mathrm{~d} y
\end{aligned}
$$

where, the integrand on the right-hand side is zero in an unyielded region of the flow, i.e., $\eta \rightarrow \infty$ in such regions. It follows that the vector of averaged velocities $(\bar{v}, \bar{w})$ is parallel to the $(\varphi, \xi)$ plane to the vector $\vec{G}$ : 
$\vec{G}=\left(-\frac{1}{r_{a}} \frac{\partial p}{\partial \varphi}+\frac{\pi \Delta \rho r \sin \pi \varphi \sin a}{S t^{*}},-\frac{\partial p}{\partial \xi}-\frac{\Delta \rho\left(r_{o}-r \cos \pi \varphi\right) \cos a}{2 r_{o} \cdot S t^{*}}\right)$

This is the vector of modified pressure gradient.

Assuming that the flow is simply a Poiseuille flow in the Hele-Shaw physical model, we can establish the relationship between pressure drop and flow rate which is represented by the stream function $\Psi$ :

$-r_{a} G_{\varphi}=\frac{\partial p}{\partial \varphi}-\frac{\pi r_{a} \Delta \rho r \sin \pi \varphi \sin a}{S t^{*}}=\frac{r_{a}\left(\chi\left(\left|\nabla_{a} \Psi\right|\right)+\tau_{Y} / H\right)}{\left|\nabla_{a} \Psi\right|} \frac{\partial \Psi}{\partial \xi}$

$G_{\xi}=-\frac{\partial p}{\partial \xi}-\frac{\Delta \rho\left(r_{o}-r \cos \pi \varphi\right) \cos a}{2 r_{o} \cdot S t^{*}}=\frac{\left(\chi\left(\left|\nabla_{a} \Psi\right|\right)+\tau_{Y} / H\right)}{\left|\nabla_{a} \Psi\right|} \frac{1}{r_{a}} \frac{\partial \Psi}{\partial \varphi}$

We set $\vec{S}=\left(r_{a} G_{\xi},-r_{a} G_{\varphi}\right)$, which can describe the relationship between the stress and flow rate of fluid on the displacement interface. Finally, we obtain that:

$\vec{S}=\left(-r_{a} \frac{\partial p}{\partial \xi}-\frac{r_{a} \Delta \rho\left(r_{o}-r \cos \pi \varphi\right) \cos a}{2 r_{o} \cdot S t^{*}}, \frac{\partial p}{\partial \varphi}-\frac{\pi r_{a} \Delta \rho r \sin \pi \varphi \sin a}{S t^{*}}\right)$

where, $\mathrm{S} t^{*}$ represents the Stoke number, which is defined as $S t^{*}=\tau^{*} / \rho^{*} g^{*} H^{*}$. $r_{a}$ represents the dimensionless mean radium in the inclined well annulus. $p$ is the dimensionless pressure. $\Delta \rho$ is the dimensionless density difference. $r_{0}$ is the dimensionless wellbore radium. $r$ represents the dimensionless distance of any point $A$ on the displacement interface from wellbore center. $\tau^{*}$ represents the larger shear stress of slurry and drilling fluid. $\rho^{*}$ represents the larger density of slurry and drilling fluid. $H^{*}$ is the mean half-gap width, $\mathrm{m} . H$ is the dimensionless mean half-gap width, which is defined as $H=r_{a}(1+e \cos \pi \varphi)$.

The continuity equation is satisfied using a stream function:

$$
\frac{\partial \Psi}{\partial \varphi}=r_{a} H \bar{w}, \quad-\frac{\partial \Psi}{\partial \xi}=H \bar{v}
$$

where $\bar{v}$ and $\bar{w}$ are the dimensionless averaged velocities in the azimuthal and axial directions, respectively. $\Psi$ is a stream function.

When satisfying $|\vec{S}| \geq \frac{r_{a} \tau_{Y}}{H}$, the displacing fluid maintains flowing. We can obtain the $\vec{S}$ expressed by stream function $\Psi$ :

$$
\begin{aligned}
& \vec{S}=\left[\frac{r_{a} \chi_{K}\left(\left|\nabla_{a} \Psi\right|\right)}{\left|\nabla_{a} \Psi\right|}+\frac{r_{a} \tau_{K, Y}}{H\left|\nabla_{a} \Psi\right|}\right]_{2}^{1}\left(\frac{1}{r_{a}} \frac{\partial \Psi}{\partial \varphi}, \frac{\partial \Psi}{\partial \xi}\right) \\
& =\left\{\frac{\partial \Psi}{\partial \varphi}\left[\frac{\chi_{K}\left(\left|\nabla_{a} \Psi\right|\right)}{\left|\nabla_{a} \Psi\right|}+\frac{\tau_{K, Y}}{H\left|\nabla_{a} \Psi\right|}\right]_{2}^{1}, \frac{\partial \Psi}{\partial \xi}\left[\frac{r_{a} \chi_{K}\left(\left|\nabla_{a} \Psi\right|\right)}{\left|\nabla_{a} \Psi\right|}+\frac{r_{a} \tau_{K, Y}}{H\left|\nabla_{a} \Psi\right|}\right]_{2}^{1}\right\}
\end{aligned}
$$

where, $\left|\nabla_{a} \Psi\right|=\frac{H^{m_{K}+2}}{\kappa_{K}^{m_{K}}\left(m_{K}+2\right)} \frac{\chi^{m_{K}+1}}{\left(\chi_{K}+\tau_{K, Y} / H\right)^{2}}\left[\chi_{K}+\frac{\left(m_{K}+2\right) \tau_{K, Y}}{\left(m_{K}+1\right) H}\right] \quad \chi_{K}>0$

$[x]_{2}^{1}=x_{1}-x_{2}$

where $K$ represents fluids' types, 1 is the slurry and 2 is the drilling fluid. $\chi$ denotes the excess of pressure gradient over the critical value $\tau_{K, Y} / H . \tau_{K, Y}$ represents the dimensionless yield stress of fluid $K . m_{K}$ represents the power-law index of fluid $K . \kappa_{K}$ is the dimensionless consistency of fluid $K$.

If smoothness of the interface is assumed, the tangential derivative of $p$ along the interface will also be continuous:

$\left[\frac{1}{r_{a}} \frac{\partial p}{\partial \varphi}+\frac{\partial p}{\partial \xi} \frac{\partial h}{\partial \varphi}\right]_{2}^{1}=0$

Equation (17) allows us to use (12) and (13) to eliminate the pressure, i.e.,

$$
\begin{aligned}
& {\left[\frac{\chi_{1}\left(\left|\nabla_{a} \Psi\right|\right)+\tau_{1, Y} / H}{\left|\nabla_{a} \Psi\right|}-\frac{\chi_{2}\left(\left|\nabla_{a} \Psi\right|\right)+\tau_{2, Y} / H}{\left|\nabla_{a} \Psi\right|}\right]\left(\frac{\partial \Psi}{\partial \xi}-\frac{1}{r_{a}} \frac{\partial \Psi}{\partial \varphi} \frac{\partial h}{\partial \varphi}\right)+} \\
& \frac{\pi}{S t^{*}} \Delta \rho r \sin \pi \varphi \sin a-\frac{\cos a}{2 r_{o} S t^{*}}\left[\Delta \rho\left(r_{o}-r \cos \pi \varphi\right)\right] \frac{\partial h}{\partial \varphi}=0
\end{aligned}
$$

where $h(\varphi, r, t)$ represents the kinematic condition for the interface.

\section{THE MODIFIED MODEL FOR STEADY DISPLACEMENT INTERFACE SHAPE IN INCLINED WELLS WITH CONCENTRIC ANNULI}

\subsection{Description of Fluids' Positions on the Displacement Interface}

Fluids of each position on the displacement interface are driven by different forces, especially on the wellbore low side where fluid is under greater driving force caused by density difference. As a result, local advance of the displacement interface happens. In order to describe the fluids' motion on the interface exactly, i.e., the interface evolution, we introduce a kinematic equation. After applying scaling arguments and averaging across the gap, we obtain the kinematic condition for the interface, denoted by $\xi=h(\varphi, r, t)$, as follows:

$\frac{\partial h}{\partial t}+\frac{\bar{v}}{r_{a}} \frac{\partial h}{\partial \varphi}=\bar{w}$

$h(\varphi, r, t)=g(\varphi, r)+\bar{w} t$

From Equ. (19), we can see the positions of fluids' on the displacement interface depend on two parts: (1) Distance that the entire interface moves. In time $t$, fluids' positions change $\bar{w} t$. (2) Interface changes itself in the axial direction because of the driving force caused by density difference. We express the variation of fluids' positions as $g(\varphi, r)$. In our research, 
$g(\varphi, r)$ can reflect the interface shape. Greater absolute value of $g(\varphi, r)$ means the displacement interface is longer. It is well known that the intermixing extent of two-phase fluids (slurry and drilling fluid) becomes more severe, which results in worse displacement effect. However, $g(\varphi, r)$ will be unchangeable until the steady displacement arrives.

\subsection{Establishment of the Modified Steady Displacement Interface Shape Model}

Assuming that the well is uniform in the $\xi$-direction and that a constant flow rate is pumped, we get $r_{a}=\bar{w}=1$. Whilst for the displacement interface tracking, we introduce a frame of reference $(z, \varphi)$ where $z=\xi-t$ is moving with the mean speed of the flow, (=unity). The interface separates slurry, the lower displacing fluid, from drilling mud. We consider a long domain $z \in(-L, L)$, with the steady interface centred at $z=0$. We denote the interface position in the moving frame by:

$z=g(\varphi, r)=h(\varphi, r, t)-t$

and write the stream function $\Psi$ as:

$\Psi=\Phi+\varphi+\frac{e}{\pi} \sin \pi \varphi$

i.e., $\Phi$ is the stream function in the moving frame. Equation (18) allows us to use (20) and (21) to eliminate $\frac{\partial \Psi}{\partial \varphi}, \frac{\partial \Psi}{\partial \xi}$ and $\frac{\partial h}{\partial \varphi}$. Finally, we obtain the continuity condition at the interface:

$$
\begin{aligned}
& {\left[\frac{\chi_{1}\left(\left|\nabla_{a}\left(\Phi+\varphi+\frac{e}{\pi} \sin \pi \varphi\right)\right|\right)+\tau_{1, Y} / H}{\left|\nabla_{a}\left(\Phi+\varphi+\frac{e}{\pi} \sin \pi \varphi\right)\right|}-\right.} \\
& {\left[\frac{\chi_{2}\left(\left|\nabla_{a}\left(\Phi+\varphi+\frac{e}{\pi} \sin \pi \varphi\right)\right|\right)+\tau_{2, Y} / H}{\left|\nabla_{a}\left(\Phi+\varphi+\frac{e}{\pi} \sin \pi \varphi\right)\right|}\right.} \\
& \times\left[\frac{\partial \Phi}{\partial z}-\left(\frac{\partial \Phi}{\partial \varphi}+1+e \cos \pi \varphi\right) \frac{\partial g}{\partial \varphi}\right] \\
& -\frac{1}{S t^{*}} \frac{\Delta \rho\left(r_{o}-r \cos \pi \varphi\right) \cos a}{2 r_{o}} \frac{\partial g}{\partial \varphi}= \\
& -\frac{1}{\pi} \frac{\Delta \rho r \sin \pi \varphi \sin a}{S t^{*}}
\end{aligned}
$$

Setting $\Phi=0$ and $e=0$ (concentric annulus) gives that $\Psi=\varphi$. Hence $\left|\nabla_{a} \varphi\right|=1$, we simplify Equ. (22), integrate it for $\varphi$ and set $g(\pi / 2, r)$ to zero. Finally, the displacement interface shape is obtained: $g(\varphi, r)=2 r_{o} \tan a \ln \left[\frac{A+\frac{\cos a}{2 r_{o} \cdot S t^{*}} \Delta \rho\left(r_{o}-r \cos \pi \varphi\right)}{A+\frac{\cos a}{2 r_{o} \cdot S t^{*}} \Delta \rho r_{o}}\right]$

where: $A=\left[\chi_{K}(1)+\frac{\tau_{K, Y}}{H}\right]_{2}^{1}$

\section{THE MODIFIED MODEL FOR STEADY DISPLACEMENT INTERFACE SHAPE IN INCLINED WELLS WITH ECCENTRIC ANNULI}

In this section, we show that it is also possible to derive an analytical solution for Equ. (22) in the case that $e \neq 0$ (eccentric annulus) by means of a regular perturbation expansion. When applying this method, casing's concentricity is regarded as an equilibrium state. Then we give the casing a slight perturbation to make it deviate from the equilibrium and reach an eccentric state finally. By solving the perturbation term we can get some information about the eccentric state.

Establishment process of the interface shape model in an eccentric annulus is as follows: Two variables of stream function $\Phi(z, \varphi)$ and interface shape $g(\varphi, r)$ in the steady displacement are expanded into asymptotic series respectively. The asymptotic series expressions are with respect to perturbation term $e$. Then we neglect the higherorder (more than one) term for $e$ in asymptotic series expansion and get two linear functions. Finally, we substitute two linear functions into the constraint condition (Equ. 22) that the stream function and interface shape satisfy. With the method of separation of variables and integration for $\partial g / \partial \varphi$ , the steady interface shape model in an eccentric annulus can be obtained.

The partial derivative of interface shape $g(\varphi, r)$ in concentric annulus for $\varphi$ is given by:

$\frac{\partial g}{\partial \varphi}=\frac{\left(\pi / S t^{*}\right) \cdot \Delta \rho r \sin \pi \varphi \sin a}{\left[\chi_{K}(1)+\tau_{K, Y} / H\right]_{2}^{1}+\frac{\cos a}{2 r_{o} \cdot S t^{*}} \cdot \Delta \rho\left(r_{o}-r \cos \pi \varphi\right)}$

From the above Equ. (24), if $\left[\chi_{K}(1)+\tau_{K, Y} / H\right]_{2}^{1} \gg \Delta \rho / S t^{*}$ in the denominator, the value $(\partial g / \partial \varphi) \rightarrow 0$. We can impose that the requirement of the zeroth order steady state shape is $O(e)$. This requirement is equivalent to assuming that:

$\frac{\left(\pi / S t^{*}\right) \cdot \Delta \rho r \sin \pi \varphi \sin a}{\left[\chi_{K}(1)+\tau_{K, Y} / H\right]_{2}^{1}+\frac{\cos a}{2 r_{o} \cdot S t^{*}} \cdot \Delta \rho\left(r_{o}-r \cos \pi \varphi\right)}=O(e)$

The asymptotic series expansion of stream function $\Phi(z, \varphi)$ and interface shape $g(\varphi, r)$ with respect to eccentricity $e$ is given respectively by: 
$\Phi(z, \varphi)=e \Phi_{1}(z, \varphi)+e^{2} \Phi_{2}(z, \varphi)+\cdots$

$g(\varphi, r)=e g_{1}(\varphi, r)+e^{2} g_{2}(\varphi, r)+\cdots$

We substitute Equ. (26) and (27) after neglecting the higher-order term into (22) and linearize it, to give:

$$
\begin{aligned}
0= & e\left[\begin{array}{l}
\left(\chi_{1}(1,1)+\tau_{1, Y}-\chi_{2}(1,1)-\tau_{2, Y}\right)\left(\frac{\partial \Phi_{1}}{\partial z}(\varphi, 0)-\frac{\partial g_{1}}{\partial \varphi}\right) \\
-\frac{1}{S t^{*}} \frac{\Delta \rho\left(r_{0}-r \cos \pi \varphi\right) \cos a}{2 r_{0}} \frac{\partial g_{1}}{\partial \varphi}
\end{array}\right] \\
& +\frac{1}{\pi} \frac{\Delta \rho r \sin \pi \varphi \sin a}{S t^{*}}+O\left(e^{2}\right)
\end{aligned}
$$

Note that, because of the zeroth order $O(e)$, both terms above have the same order. Rearranging we have:

$$
\begin{gathered}
\frac{\partial g_{1}}{\partial \varphi}=\frac{1}{e} \frac{\left(\pi / S t^{*}\right) \cdot \Delta \rho r \cos \pi \varphi \sin a}{\left[\chi_{K}(1)+\tau_{K, Y} / H\right]_{2}^{1}+\frac{\cos a}{2 r_{o} \cdot S t^{*}} \cdot \Delta \rho\left(r_{o}-r \cos \pi \varphi\right)} \\
+\frac{\left[\left(\chi_{K}(1,1)+\tau_{K, Y} / H\right)\right]_{2}^{1} \frac{\partial \Phi_{1}}{\partial z}(\varphi, 0)}{\left[\chi_{K}(1)+\tau_{K, Y} / H\right]_{2}^{1}+\frac{\cos a}{2 r_{o} \cdot S t^{*}} \cdot \Delta \rho\left(r_{o}-r \cos \pi \varphi\right)}
\end{gathered}
$$

Equ. (29) indicates that the first term is simply the concentric annular solution. The second term represents the effect of eccentricity.

where:

$\Phi_{K}(\varphi, z)=\left(1+\frac{\chi_{K, H}(1,1)-\tau_{K, Y}}{\chi_{K}^{\prime}(1,1)}\right)\left(-1+\frac{\cosh \alpha_{K}(L+z)}{\cosh \alpha_{K} L}\right) \frac{\sin \pi \varphi}{\pi} \quad(\varphi, z) \in \Omega_{K}$

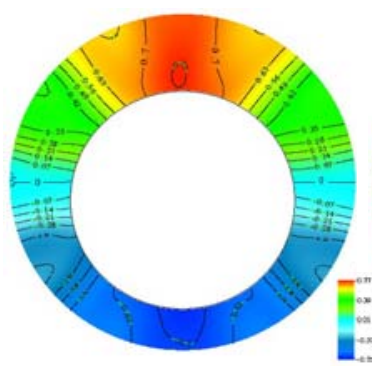

$\Delta \rho=0.1 \mathrm{~g} / \mathrm{cm}^{3}$

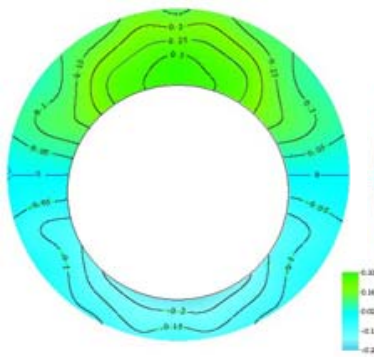

$\Delta \rho=0.5 \mathrm{~g} / \mathrm{cm}^{3}$

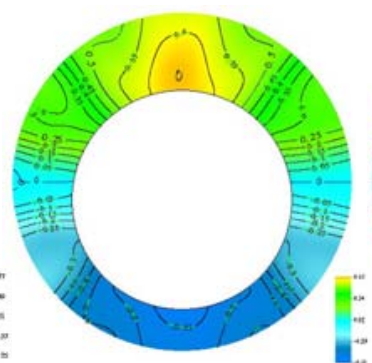

$\Delta \rho=0.2 \mathrm{~g} / \mathrm{cm}^{3}$

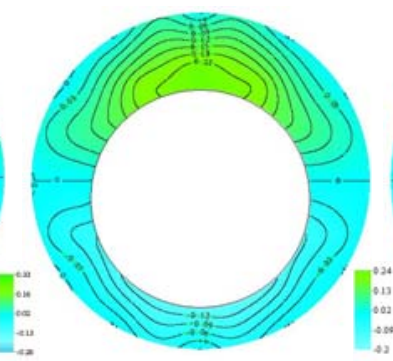

$\Delta \rho=0.6 \mathrm{~g} / \mathrm{cm}^{3}$ $\alpha_{k}^{2}=\frac{\pi^{2} \chi_{K}^{\prime}(1,1)}{\chi_{K}(1,1)+\tau_{K, Y}}>0$

According to the expression of $\left|\nabla_{a} \Psi\right|$, we can obtain the partial derivative of $\chi_{K}$ with respect to $\left|\nabla_{a} \Psi\right|$ and the partial derivative of $\chi_{K, H}$ with respect to $H$, which are given respectively by:

$$
\begin{aligned}
& \chi_{K}^{\prime}=\frac{-\left(\chi_{K}+\tau_{K, Y} / H\right)}{2\left(\chi_{K}+\tau_{K, Y} / H\right)-\frac{H^{m_{K}+2}}{k_{K}^{m_{K}}} \cdot \chi_{K}^{m_{K}+1}-\frac{H^{m_{K}+2}}{k_{K}^{m_{K}}} \cdot \frac{\tau_{K, Y}}{H} \cdot \chi_{K}^{m_{K}}} \\
& \chi_{K, H}=\frac{\frac{H^{m_{K}} \chi_{K}^{m_{K}+1} \tau_{K, Y}}{\left(m_{K}+1\right) k_{K}^{m_{K}}\left(\chi_{K}+\tau_{K, Y} / H\right)}-\frac{2 \tau_{K, Y}}{H^{2}}-\frac{\left(m_{K}+2\right)\left(\chi_{K}+\tau_{K, Y} / H\right)}{H}}{\frac{H^{m_{K}+2} \chi_{K}^{m_{K}+1}}{\left(m_{K}+2\right) k_{K}^{m_{K}}\left(\chi_{K}+\tau_{K, Y} / H\right)}+\frac{\left(m_{K}+1\right)\left(\chi_{K}+\tau_{K, Y} / H\right)}{\chi_{K}}-2}
\end{aligned}
$$

Finally, We substitute $\Phi_{1}$ into Equ. (29), simplify and integrate to give the interface position:

$$
\begin{aligned}
g(\varphi, r)= & 2 r_{o} \tan \beta \ln \left[\frac{A+\frac{\cos a}{2 r_{o} \cdot S t^{*}} \Delta \rho\left(r_{o}-r \cos \pi \varphi\right)}{A+\frac{\cos a}{2 r_{o} \cdot S t^{*}} \Delta \rho r_{o}}\right] \\
- & \ln \left[\frac{A+\frac{\cos a}{2 r_{o} \cdot S t^{*}} \Delta \rho\left(r_{o}-r \cos \pi \varphi\right)}{A+\frac{\cos a}{2 r_{o} \cdot S t^{*}} \Delta \rho r_{o}}\right] \times \\
& {\left[\frac{e}{\pi^{2}} \frac{1}{\Delta \rho r} \frac{2 r_{o} S t^{*}}{\cos a} \sum_{K=1,2} P\left(\chi_{K}, \tau_{K, Y}, m_{K}\right)\left(\chi_{K}+\tau_{K, Y}\right) \alpha_{K} \tanh \alpha_{K} L\right] }
\end{aligned}
$$

Fig. (3). Contour lines of $g(\varphi, r)$ under different density difference for $e=0.3, a=45^{\circ}$. 

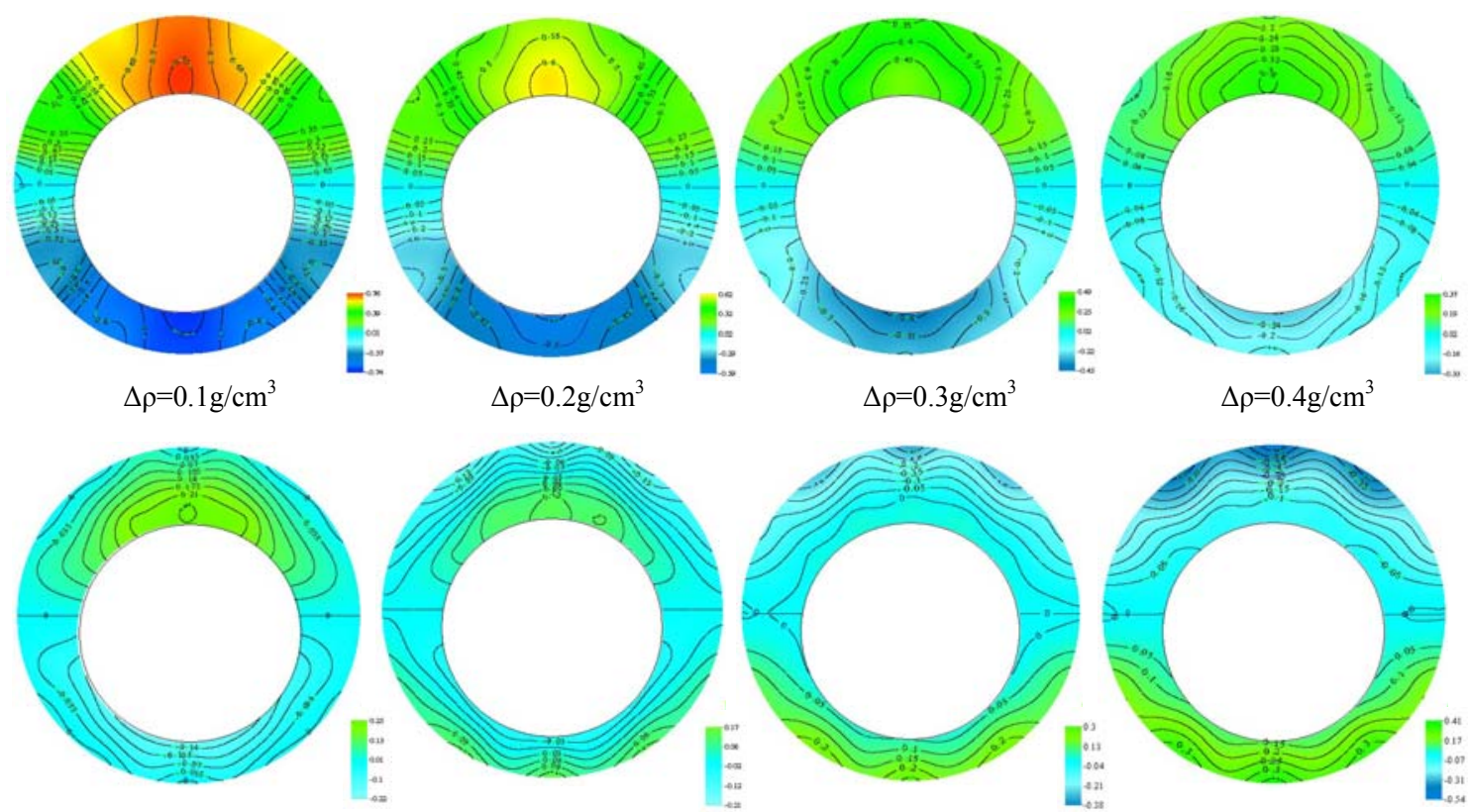

$\Delta \rho=0.5 \mathrm{~g} / \mathrm{cm}^{3}$

$\Delta \rho=0.6 \mathrm{~g} / \mathrm{cm}^{3}$

$\Delta \rho=0.7 \mathrm{~g} / \mathrm{cm}^{3}$

$\Delta \rho=0.8 \mathrm{~g} / \mathrm{cm}^{3}$

Fig. (4). Contour lines of $g(\varphi, r)$ under different density difference for $e=0.3, a=75^{\circ}$.

Table 1. Basic Parameters for Calculation

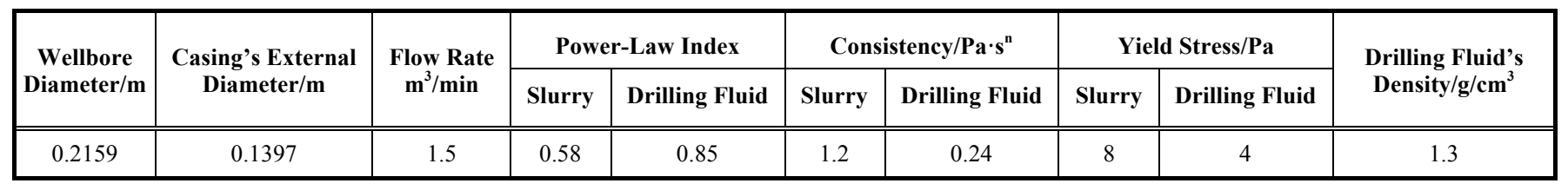

where:

$$
P\left(\chi, \tau_{Y}, m\right)=\frac{(m+1)^{2} \chi^{2}+(m+2)(2 m+1) \chi \tau_{Y}+(m+1)(m+2) \tau_{Y}^{2}}{\chi\left[(m+1) \chi+(m+2) \tau_{Y}\right]}
$$

\section{ILLUSTRATIVE RESULTS OF THE DISPLACEMENT INTERFACE SHAPE}

We take deviation angle $a=45^{\circ}$ and $a=75^{\circ}$ as examples. When calculating the displacement interface shape based on Equ. (32), for a chosen deviation angle $a$, we change the density difference from $0.1 \mathrm{~g} / \mathrm{cm}^{3}$ to $0.8 \mathrm{~g} / \mathrm{cm}^{3}$ and maintain casing's eccentricity 0.3 unchangeable. Basic parameters that we use for calculation are shown in Table $\mathbf{1}$. The calculation results are shown in Figs. $(\mathbf{3}, \mathbf{4})$.

As shown in Fig. (3) and Fig. (4), $g(\varphi, r)$ is changing with annular azimuthal angle and radium. Because of setting $g(\pi / 2, r)$ to zero, $g(\varphi, r)$ has both positive and negative values. In our research, positive $g(\varphi, r)$ represents the interface here which moves ahead of that in the annulus center, otherwise, behind the center interface. It is certain that larger absolute value of $g(\varphi, r)$ means the interface here is further away from that in the annulus center. We define $g(\varphi, r)_{\max }$ as the maximum $g(\varphi, r)$, which represents the interface front and $g(\varphi, r)_{\min }$ is the minimum $g(\varphi, r)$, which represents the interface trailing edge. There is no doubt that $g(\varphi, r)_{\max ^{-}} g(\varphi, r)_{\min }$ is the entire displacement interface length. It is known that a longer interface means more displacing fluid becomes contaminated. When the interface becomes too long, it might be that the trailing edge of the interface fails to reach a designed cementing segment at the end of cementing operation, which reduces displacement effectiveness and adversely affects cementing quality and well integrity. Compared to $g(\varphi, r)$ under different density differences, we can find:

(1) At each point of annulus, there exists a big difference among $g(\varphi, r)$ although the annular azimuthal angle is equal. We conclude that the displacement interface shape is not only related to azimuthal angle but also to the distance of fluids from the wellbore center, which is not involved in previous literatures.

When the density difference is smaller, the driving force cannot overcome the resistance in the annular narrow side, which results in slurry advancing in the annular wide side. The position of $g(\varphi, r)_{\max }$ is on the external casing wall of the annular wide side. However, when the density difference is greater, the opposite results will occur. The position of $g(\varphi, r)_{\max }$ is on the wellbore wall of the annular narrow side.

(3) The displacement interface length in inclined wells with eccentric annuli is decreased firstly and then increased with the increase of density difference. The 
extent and position of slurry' advancing depends on the combined effect of density difference and casing's eccentricity. Because the effects of density difference and casing's eccentricity on the interface shape are opposite, there exists an optimal density difference which can make the interface length minimum.

The optimal density differences for $a=45^{\circ}$ and $a=45^{\circ}$ are $0.7 \mathrm{~g} / \mathrm{cm}^{3}$ and $0.5 \mathrm{~g} / \mathrm{cm}^{3}$ respectively. It can be seen that the optimal density difference in inclined well gradually decreased with deviation angle. Therefore, a greater density difference can obtain better displacement effect for vertical wells when meeting the conditions of pressure stability and anti-leak. Otherwise, a smaller density difference can get better displacement effect for horizontal wells.

\section{CONCLUSIONS}

In this paper, by comprehensively analyzing the effects of both azimuthal angle and annular radium on the displacement interface shape, we establish a modified model for steady displacement interface shape in inclined wells with eccentric annuli, which will be more suitable for describing the actual displacement interface shape in cementing. Based on this model, we can analyze the change of displacement interface shape with deviation angle, casing eccentricity and density difference; moreover, determine what conditions make the interface length minimum. This model provides a basis for optimization design of displacing fluids' parameters during inclined well cementing.

According to our research, we can conclude that the position of slurry fingering and the intermixing extent of two-phase fluids depend on the relative effects between casing eccentricity and density difference. A smaller density difference obviously results in slurry advancing in the annular wide side. The position of interface front is on the external casing wall of annular wide side. Otherwise, a greater density difference causes slurry advancing in annular narrow side and the position of interface front is on the wellbore wall of the annular narrow side. The opposite results show that the displacement interface length is decreasing firstly and then increased with the increase of density difference. There does exist an optimal density difference to make the interface length minimum.

For a certain casing's eccentricity, the optimal density difference in inclined wells is gradually decreased with deviation angle. When meeting the conditions of pressure stability and anti-leak, greater density difference can obtain better displacement effect for vertical wells, otherwise, smaller density difference can be good for horizontal wells.

\section{CONFLICT OF INTEREST}

The authors confirm that this article content has no conflicts of interest.

\section{ACKNOWLEDGEMENTS}

The paper is supported by Natural Science Foundation of Heilongjiang Province of China (Project No. QC2012C021).

\section{REFERENCES}

[1] Shu, Q.G.; Luo D.M.; Jiao J.F. Theory and technology on cementing displacement in irregular well bore annulus across shale section. Drill. Product. Technol., 2012, 35(4), 29-31.

[2] Liu, C.G. Key technology research and application of cementing in horizontal wells of unconventional oil and gas reservoir. Oil Drill. Product. Technol., 2013, 35(2), 48-51.

[3] Li, M.Z.; Wang, C.W.; Wang, C.Q. Numerical simulation of cement displacement in eccentric annulus at highly deviated well. Petrol. Drill. Techniq., 2012, 40(5), 40-44.

[4] Zhang, S.J.; Xue, L.; Wang, Z.M. Numerical similation study on the influence law of density difference on cementing displacement interface in the horizontal well. Drill. Product. Technol., 2012, 35(6), 15-17.

[5] Bittleston, S.H.; Ferguson, J.; Frigaard, I.A. Mud removal and cement placement during primary cementing of an oil well: Laminar nonNewtonian displacements in an eccentric annular Hele-Shaw cell. $J$. Eng. Mathemat., 2002, 13, 229-253.

[6] Frigaard, I.A.; Pelipenko, S. Effect of density difference on displacement efficiency during low-rate cementing. Petrol. Drill. Techniq., 2007, 35, 79-82

[7] Pelipenko, S.; Frigaard, I.A. Visco-plastic fluid displacements in near vertical narrow eccentric annuli: prediction of travelling-wave solutions and interfacial in-stability. J. Fluid. Mech., 2004, 1-35.

[8] Pelipenko, S.; Frigaard, I.A. Mud removal and cement placement during primary cementing of an oil well. J. Eng. Math., 2004, 48, 1-26.

[9] Pelipenko, S.; Frigaard, I.A. Two-dimensional computational simulation of eccentric annular cementing displacements. IMA J. Appl. Math., 2004, 69, 557-583.

[10] Miguel, A.; Moyers-Gonzalez, M.A.; Frigaard I.A. Kinematic instabilities in two-layer eccentric annular flows, part 1: Newtonian fluids. J. Eng. Math., 2007, 62, 103-131.

[11] Moyers-Gonzalez, M.A.; Frigaard, I.A. Kinematic instabilities in twolayer eccentric annular flows, part 2: shear-thinning and yield-stress effects. J. Eng. Math., 2009, 65, 25-52.

[12] Carrasco-Teja, M.; Frigaard, I.A.; Seymour, B.R.; Storey S. Viscoplastic fluid displacements in horizontal narrow eccentric annuli: stratification and travelling wave solutions. J. Fluid Mech., 2008, 605, 293-327.

[13] Carrasco-Teja, M.; Frigaard, I.A.; Seymour, B. Cementing horizontal wells: Complete zonal isolation without casing Rotation. CIPC/SPE Gas Technology Symposium 2008 Joint Conference, 2008, 2-6.

[14] Malekmohammadi, S.; Carrasco-Teja, M.; Storey, S., Frigaard, I.A.; Artinez, D.M. An experimental study of laminar displacement flows in narrow vertical eccentric annuli. J. Fluid Mech., 2010, 649, 371-398.

This is an open access article licensed under the terms of the Creative Commons Attribution Non-Commercial License (http://creativecommons.org/licenses/by-nc/3.0/) which permits unrestricted, non-commercial use, distribution and reproduction in any medium, provided the work is properly cited. 